\title{
New Ester and Furocoumarins from the Roots of Pituranthos tortuosus
}

\author{
Maged S. Abdel-Kader* \\ Department of Pharmacognosy, College of Pharmacy, University of Alexandria, Alexandria, Egypt 21215
}

\begin{abstract}
Sete compostos foram isolados, a partir das frações solúveis em $\mathrm{CHCl}_{3}$, de raízes de Pituranthos tortuosos; adicionalmente manitol foi cristalizado a partir do extrato alcoólico. Usando diferentes técnicas espectroscópicas os compostos isolados foram identificados como bergaptana, graveolana, xanthotoxinona, isopimpinela, dimetoxi aesculetina, 3-O- $\beta$-glicopiranosil estigmasterol e também o novo éster umbelato de 4-metoxifenila. A estrutura do novo éster foi confirmada pela síntese completa e se mostrou inativo a testes antimicrobiais e de citotoxicidade.
\end{abstract}

Seven compounds were isolated from the $\mathrm{CHCl}_{3}$ soluble fraction of the roots of Pituranthos tortuosus; in addition, mannitol was crystallized out of the total alcoholic extract. Using different spectroscopic techniques, the isolated compounds were identified as bergapten, graveolone, xanthotoxin, isopimpinellin, aesculetin dimethyl ether, stigmasterol glucoside, in addition to the new ester 4-methoxyphenylumbellate. The structure of the new ester was confirmed by total synthesis and it was found to be inactive in antimicrobial and cytotoxicity assays.

Keywords: Pituranthos tortuosus, Apiaceae, 4-methoxyphenylumbellate, furanocoumarins, mannitol, stigmasterol glucoside, synthesis

\section{Introduction}

Members of the family Apiaceae (Umbelliferae) are well known producers of furanocoumarins. In this regard, Apiaceae is in the first place followed by Rutaceae and Moraceae. ${ }^{1}$ Furanocoumarins have several interesting biological activities, such as analgesic, antiinflammatory, antibacterial, antiviral, anticoagulant, in addition to their well known photosensitizing effect. ${ }^{2-8}$ In Egypt, the genus Pituranthos (Deverra) is represented by two species. ${ }^{9}$ While five furanocoumarins were isolated from $P$. triradiatus, the aerial parts of $P$. tortuosus was found to be free from these compounds..$^{10}$ This result intiated the study of the plant roots for the presence of furanocoumarins since they are taxonomic markers for the family.

\section{Results and Discussions}

Spectral data indicated that compounds $\mathbf{1}$ and $\mathbf{3}$ are furanocoumarins with a methoxyl group at either C-5 or C-8. The position of the methoxyl was assigned based on comparison of the ${ }^{13} \mathrm{C}$-NMR data with those in the literature $^{11}$. The negative CIMS data of $\mathbf{1}$ (bergapten)

* e-mail: mpharm101@ hotmail.com showed a base peak at $\mathrm{m} / \mathrm{z} 201$ resulting from the fission of the aromatic ether bond indicating a 1,3,5 oxygenation of the aromatic ring which stabilizes the resulting phenoxide anion. ${ }^{12}$ That ion in $\mathbf{3}$ (xanthotoxin) was of very low intensity indicating C-8 oxygenation.

Compounds 2, 4 and 5 were identified as graveolone, ${ }^{13-15}$ isopimpinellin ${ }^{16}$ and aesculetin dimethyl ether, ${ }^{17}$ respectively, by comparison of their data with those of the literature. However, the ${ }^{13} \mathrm{C}-\mathrm{NMR}$ data for the linear dihydrobenzodipyrandione; graveolone (2) is reported here for the first time. Graveolone is a compound of very limited occurrence and has only been isolated from dill and parsley. ${ }^{13,16}$

Compound 6 gives a positive reaction with $\mathrm{FeCl}_{3}$ indicating the presence of free phenolic $\mathrm{OH}$ group(s). In the ${ }^{1} \mathrm{H}-\mathrm{NMR}$ (Table 1), the ABX system (6.33, dd, $J$ 2.2, 9.0 $\mathrm{Hz} ; 6.31, \mathrm{~d}, J 2.2 \mathrm{~Hz} ; 7.38, \mathrm{~d}, J 9.0 \mathrm{~Hz}$ ) was assigned for a trisubstituted aromatic system. The chemical shifts of the protons and carbons supported a 1, 2, 4-trisubstitution with two oxygenations at 2,4 rather than 1,3 , 4trisubstitutions. ${ }^{18-21}$ Two other doublets $(J 16.0 \mathrm{~Hz})$ each integrated for 1 proton at $\delta 6.58$ and 8.03 with their correlated carbons (Table 1) were assigned to trans oriented conjugated vinyl protons. The substituted aromatic system along with the vinyl protons as well as the carbonyl signal at $167.91 \mathrm{ppm}$ in the ${ }^{13} \mathrm{C}$-NMR (Table 1) were assigned for a 2, 4-dioxygenated cinnamate moiety. 
<smiles>[R]c1c2ccoc2c([R])c2oc(=O)ccc12</smiles><smiles>CC1(C)CC(=O)c2cc3ccc(=O)oc3cc2O1</smiles>

1: $\mathrm{R}^{1}=\mathrm{OCH}_{3} \quad \mathrm{R}^{2}=\mathrm{H}$ 3: $\mathrm{R}^{1}=\mathrm{H} \quad \mathrm{R}^{2}=\mathrm{OCH}_{3}$ 4: $\mathrm{R}^{1}=\mathrm{R}^{2}=\mathrm{OCH}_{3}$<smiles>COc1ccc(OC(=O)/C=C/c2ccc(O)cc2O)cc1</smiles>

Both EIMS ( $\mathbf{M}^{+}$at $\left.m / z, 286\right)$ and CIMS $\left(\mathbf{M}^{+}+1\right.$ at $m / z$ 287) (see experimental) were consistent with the molecular formula $\mathrm{C}_{16} \mathrm{H}_{14} \mathrm{O}_{5}$. Fourteen carbon signals were observed in the ${ }^{13} \mathrm{C}$-NMR spectrum (Table 1). However, two of these signals (114.18 and $122.41 \mathrm{ppm}$ ) correlated by an HMQC experiment to two doublets at $\delta 6.92(2 \mathrm{H}, J 8.8 \mathrm{~Hz})$ and $7.03(2 \mathrm{H}, \mathrm{d}, J 8.8 \mathrm{~Hz})$ were assigned for a $p$-dioxygenated benzene ring. The position of the $\mathrm{OCH}_{3}(\delta 3.77,54.85$ ppm in ${ }^{1} \mathrm{H}$ - and ${ }^{13} \mathrm{C}-\mathrm{NMR}$ respectively) at $\mathrm{C}-4$ ' was determined by a GOESY experiment where irradiation of the $\mathrm{OCH}_{3}$ signal resulted in an enhancement in the doublet at $\delta 6.92(2 \mathrm{H}, J 8.8 \mathrm{~Hz})$.

The above discussion indicated that $\mathbf{6}$ is an ester of umbellic acid (2, 4-dihydroxy cinnamic acid) with 4methoxyphenol.
As final proof, 6 was obtained by total synthesis (Figure 1). The commercially available umbelliferone (Aldrich) was treated with $5 \%$ alcoholic solution of $\mathrm{NaOH}$ to open the lactone ring. This reaction resulted in the formation of umbellic acid (2, 4-dihydroxy trans-cinnamic acid) 7 which was protected by TBDMSCl to give 7a. Protected umbellic acid 7a was then coupled with 4-methoxyphenol to produce 6a. Deprotection of 6a gave $\mathbf{6}$. Umbellic acid is a possible precursor for umbelliferone which is the key compound in the biosynthesis of furanocoumarins. ${ }^{22}$ The isolation of compounds 1- $\mathbf{6}$ from the roots of $P$. tortuosus indicated that its biosynthetic pathway is consistent with those in other members of the family Apiaceae.

Compound $\mathbf{6}$ was inactive when tested against A2780 (Human Ovarian cancer cell), Escherichia coli, Staphylococcus albus and Candida albicans.

\section{Experimental}

\section{General procedure}

Melting points were determined using Kofler's hot stage instrument and are uncorrected. UV spectra were determined using UV-1201 Shimadzu spectrometer. NMR spectra were recorded on a Varian Unity 400 NMR instrument at $399.951 \mathrm{MHz}$ for ${ }^{1} \mathrm{H}$ and $100.578 \mathrm{MHz}$ for ${ }^{13} \mathrm{C}$. MS were taken on a VG 7070 E-HF. All chemicals used in the synthesis of $\mathbf{6}$ were obtained from Aldrich Chemicals Company.

Table 1. ${ }^{1} \mathrm{H}^{\mathrm{a}}{ }^{\mathrm{a}}$ and ${ }^{13} \mathrm{C}-\mathrm{NMR}$ data for compounds $\mathbf{6}, \mathbf{6 a}, \mathbf{7}$ and $\mathbf{7 a}$

\begin{tabular}{|c|c|c|c|c|c|c|c|}
\hline \multirow[t]{2}{*}{ No. } & \multicolumn{2}{|c|}{$6^{\mathbf{b}}$} & \multicolumn{2}{|c|}{$6 a^{b}$} & \multicolumn{2}{|c|}{$7^{\mathrm{c}}$} & \multirow{2}{*}{$\frac{7 \mathbf{a}^{\mathbf{b}}}{{ }^{1} \mathbf{H}}$} \\
\hline & ${ }^{1} \mathbf{H}$ & ${ }^{13} \mathrm{C}$ & ${ }^{1} \mathbf{H}$ & ${ }^{13} \mathrm{C}$ & ${ }^{1} \mathbf{H}$ & ${ }^{13} \mathrm{C}$ & \\
\hline 1 & & 113.56 & & 119.60 & & 113.81 & \\
\hline 2 & & 157.51 & & 156.41 & & 158.70 & \\
\hline 3 & $6.31 \mathrm{~d} J 2.2$ & 102.34 & $6.34 \mathrm{~d} J 2.3$ & 111.74 & $6.31 \mathrm{~m}$ & 102.33 & $6.47 \mathrm{~d} \mathrm{~J} 2.5$ \\
\hline 4 & & 159.30 & & 157.28 & & 160.90 & \\
\hline 5 & $6.33 \mathrm{dd} J 2.2,9.0$ & 107.81 & $6.51 \mathrm{dd} \quad J \quad 2.3,8.7$ & 114.53 & $6.31 \mathrm{~m}$ & 107.59 & $6.48 \mathrm{dd} J 2.5,8.7$ \\
\hline 6 & $7.38 \mathrm{~d} J 9.0$ & 130.85 & $7.49 \mathrm{~d} J 8.7$ & 128.62 & $7.31 \mathrm{~d} J 9.0$ & 130.22 & $7.44 \mathrm{~d} J 8.7$ \\
\hline$\alpha$ & $6.58 \mathrm{~d} J 16.0$ & 143.22 & $6.45 \mathrm{~d} \quad J 16.0$ & 141.82 & $6.37 \mathrm{~d} J 15.9$ & 141.27 & $6.28 \mathrm{~d} J 16.0$ \\
\hline$\beta$ & $8.03 \mathrm{~d} J 16.0$ & 112.23 & $8.20 \mathrm{~d} \quad J 16.0$ & 114.39 & $7.86 \mathrm{~d} J 15.9$ & 114.30 & $8.10 \mathrm{~d} J 16.0$ \\
\hline $\mathrm{C}=\mathrm{O}$ & & 167.91 & & 166.61 & & 171.83 & \\
\hline 1 ' & & 144.76 & & 144.76 & & & \\
\hline 2 , & $7.03 \mathrm{~d} J 8.8$ & 122.41 & $6.91 \mathrm{~d} \quad J 8.9$ & 122.66 & & & \\
\hline 3, & $6.92 \mathrm{~d} J 8.8$ & 114.18 & $7.07 \mathrm{~d} \quad J 8.9$ & 114.64 & & & \\
\hline $4^{\prime}$ & & 161.65 & & 159.36 & & & \\
\hline 5, & $6.92 \mathrm{~d} J 8.8$ & 114.18 & $7.07 \mathrm{~d} \quad J 8.9$ & 114.64 & & & \\
\hline 6 & $7.03 \mathrm{~d} J 8.8$ & 122.41 & $6.91 \mathrm{~d} \quad J 8.9$ & 122.66 & & & \\
\hline $\mathrm{OCH}_{3}$ & $3.77 \mathrm{~s}$ & 54.85 & $3.81 \mathrm{~s}$ & 55.83 & & & \\
\hline $\mathrm{CH}_{3}$ & & & $0.23 \mathrm{~s}, 2 \mathrm{CH}_{3}$ & $-4.112 \mathrm{CH}_{3}$ & & & $0.22 \mathrm{~s}, 2 \mathrm{CH}_{3}$ \\
\hline & & & $0.25 \mathrm{~s}, 2 \mathrm{CH}_{3}$ & $-4.032 \mathrm{CH}_{3}$ & & & $0.24 \mathrm{~s}, 2 \mathrm{CH}_{3}$ \\
\hline & & & $0.99 \mathrm{~s}, 3 \mathrm{CH}_{3}$ & $25.843 \mathrm{CH}_{3}$ & & & $0.98 \mathrm{~s}, 3 \mathrm{CH}_{3}$ \\
\hline & & & $1.03 \mathrm{~s}, 3 \mathrm{CH}_{3}$ & $25.973 \mathrm{CH}_{3}$ & & & $1.04 \mathrm{~s}, 3 \mathrm{CH}_{3}$ \\
\hline
\end{tabular}

\footnotetext{
a $J$ values in $\mathrm{Hz}$; ${ }^{\text {b }}$ Spectra were measured in $\mathrm{CDCl}_{3}$; ${ }^{\mathrm{c}}$ Spectra were measured in $\mathrm{CD}_{3} \mathrm{OD}$.
} 


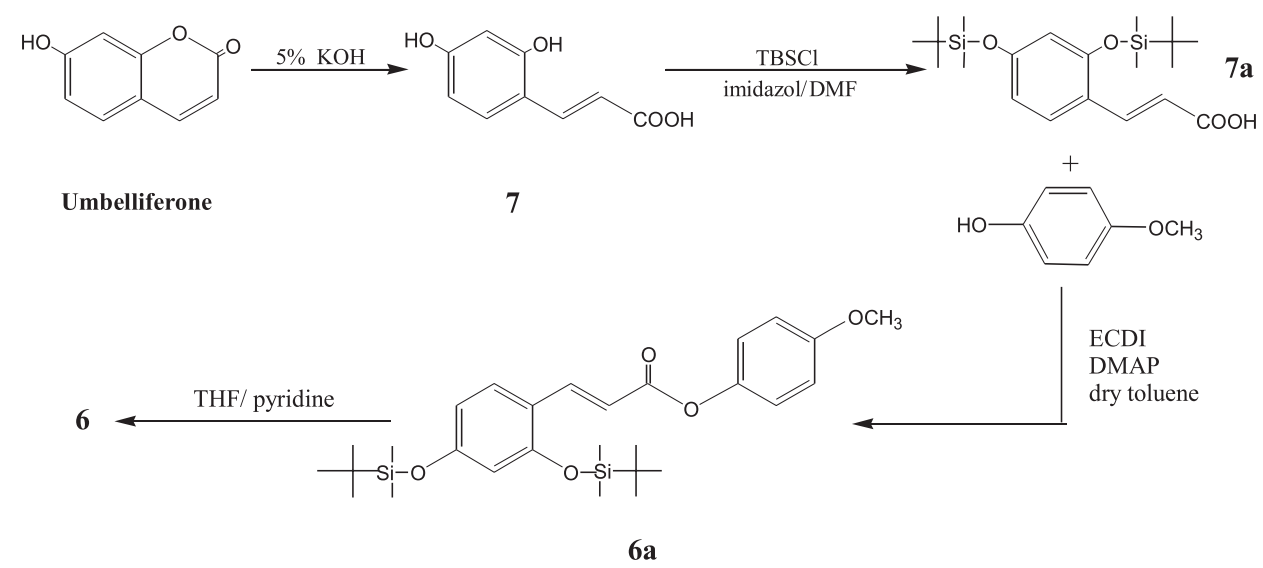

Figure 1. Scheme for synthesis of 6 from umbelliferone.

\section{Plant material}

Roots of Pituranthos tortuosus (Desf.) Benth. were collected on April 3, 2000 from Borg El-Arab, Alexandria, Egypt. The plant was identified by Dr Sanyia Kamal, Department of Botany, Faculty of Science, University of Alexandria. A voucher specimen MS-7 is deposited in the Pharmacognosy Department, Faculty of Pharmacy, University of Alexandria, Egypt.

\section{Extraction and isolation}

$1.2 \mathrm{~kg}$ of the dried roots was extracted with $\mathrm{MeOH}(6 \mathrm{~L})$. The methanolic extract was concentrated to $200 \mathrm{~mL}$. At that point $400 \mathrm{mg}$ of colourless crystals of mannitol were separated out of the solution. The concentrated methanolic solution was diluted with water to $300 \mathrm{~mL}$ and extracted exhaustively with hexane $(700 \mathrm{~mL}), \mathrm{CHCl}_{3}(700 \mathrm{~mL})$ and EtOAc $(500 \mathrm{~mL})$. The $\mathrm{CHCl}_{3}$ soluble fraction ( $3 \mathrm{~g}$ ) was fractionated over silica gel column (200 g, $3 \mathrm{~cm}$ ) eluting with $1 \% \mathrm{MeOH}$ in $\mathrm{CHCl}_{3}$, with gradual increasing of the $\mathrm{MeOH}$ contents and $200 \mathrm{~mL}$ fractions were collected. Fractions 2- $4(1 \% \mathrm{MeOH}, 1.0 \mathrm{~g})$ were rechromatographed over silica gel column (100 g, 2.5 $\mathrm{cm}$ ) eluting with $25 \%$ hexane in $\mathrm{CHCl}_{3}, \mathrm{CHCl}_{3} /$ hexane and then $\mathrm{CHCl}_{3} / \mathrm{MeOH}$ mixtures. Fraction $2(50 \%$ hexane in $\mathrm{CHCl}_{3}, 150 \mathrm{mg}$ ) was subjected to repeated prep TLC using hexane/ $\mathrm{CHCl}_{3}(2: 1)$ as developing system (triple run) to afford $\mathbf{1}(8 \mathrm{mg}), \mathbf{2}(5 \mathrm{mg}), \mathbf{3}(14 \mathrm{mg}), \mathbf{4}(6 \mathrm{mg})$ and $\mathbf{5}(4 \mathrm{mg})$.

Fraction $5(2 \% \mathrm{MeOH}, 100 \mathrm{mg})$ was further purified over flash silica gel column $(30 \mathrm{~g}, 2.5 \mathrm{~cm})$ eluting with $1 \%$ $\mathrm{MeOH}$ in $\mathrm{CHCl}_{3}$. Fraction $3(7 \mathrm{mg})$ was subjected to prep TLC over $\mathrm{RP} 18$ and $\mathrm{MeOH} / \mathrm{H}_{2} \mathrm{O}$ (7: 3) as developing system to afford $2 \mathrm{mg}$ of $\mathbf{6}$.

Fraction 9 afforded $50 \mathrm{mg}$ of stigmasterol glucoside after crystallization from $\mathrm{MeOH}$.
Bergapten (5-Methoxypsoralen) (1). $\mathrm{C}_{12} \mathrm{H}_{8} \mathrm{O}_{4}, \mathrm{mp}$ 191$192{ }^{\circ} \mathrm{C}$. EIMS $m / z$ (rel. Int.): $216\left(90, \mathrm{M}^{+}\right)$. Negative CIMS $m / z$ (rel. Int.): $217\left(7, \mathbf{M}^{+}+1\right), 216\left(10, \mathbf{M}^{+}\right), 201\left(100, \mathbf{M}^{+}-\right.$ $\mathrm{CH}_{3}$ ).

Graveolone (2). $\mathrm{C}_{14} \mathrm{H}_{12} \mathrm{O}_{4}, \mathrm{mp} 176-178{ }^{\circ} \mathrm{C}$. UV $\lambda_{\max }$ ' $\mathrm{nm}(\mathrm{MeOH}) 253,303,308,329,344$. IR (film): $v_{\max } / \mathrm{cm}^{-1}$ : 1733 (lactone $\mathrm{C}=\mathrm{O}), 1688(\mathrm{C}=\mathrm{O}), 1620,1589,1542,821$. ${ }^{1} \mathrm{H}-\mathrm{NMR}\left(\mathrm{ppm}, \mathrm{CDCl}_{3}\right): \delta 1.49\left(6 \mathrm{H}, \mathrm{s}, 2 \mathrm{XCH}_{3}\right), 2.77(2 \mathrm{H}, \mathrm{s}$, H-2'), $6.30(1 \mathrm{H}, \mathrm{d}, J 9.7 \mathrm{~Hz}, \mathrm{H}-3), 6.83(1 \mathrm{H}, \mathrm{s}, \mathrm{H}-8), 7.66$ $(1 \mathrm{H}, \mathrm{d}, J 9.7 \mathrm{~Hz}, \mathrm{H}-4), 8.03(1 \mathrm{H}, \mathrm{s}, \mathrm{H}-5) .{ }^{13} \mathrm{C}-\mathrm{NMR}$ (ppm, $\left.\mathrm{CDCl}_{3}\right): \delta 26.93$ (C-4', C-5'), 48.86 (C-2'), 80.98 (C-3'), 105.83 (C-8), 113.53 (C-6), 114.82 (C-3), 117.85 (C-10), 127.60 (C-5), 143.56 (C-4), 159.49 (C-9), 160.15 (C-7), 162.61 (C-2), 191.07 (C-1'). EIMS $m / z$ (rel. Int.): 244 (43, $\left.\mathrm{M}^{+}\right), 229\left(100, \mathrm{M}^{+}-\mathrm{CH}_{3}\right), 216\left(8, \mathrm{M}^{+}-\mathrm{CO}\right), 201\left(7, \mathrm{M}^{+}-\right.$ $\left.\mathrm{CH}_{3}-\mathrm{CO}\right), 189\left(81, \mathrm{M}^{+}-\mathrm{C}_{4} \mathrm{H}_{7}\right), 188\left(40, \mathrm{M}^{+}-\mathrm{C}_{4} \mathrm{H}_{8}\right), 160$ (28), 132 (12), 104 (15), 76 (37).

Xanthotoxin (8- Methoxypsoralen) (3). $\mathrm{C}_{12} \mathrm{H}_{8} \mathrm{O}_{4}, \mathrm{mp}$ 150- $151^{\circ} \mathrm{C}$. EIMS $m / z$ (rel. Int.): $216\left(100, \mathrm{M}^{+}\right)$. Negative CIMS $m / z$ (rel. Int.):217 (16, $\left.\mathrm{M}^{+}+1\right), 216\left(100, \mathrm{M}^{+}\right), 201$ (6, $\mathrm{M}^{+}-\mathrm{CH}_{3}$ ).

Isopimpinellin (4). $\mathrm{C}_{13} \mathrm{H}_{10} \mathrm{O}_{5}$, mp 115- $117{ }^{\circ} \mathrm{C}$. EIMS $m / z$ (rel. Int.): $246\left(100, \mathrm{M}^{+}\right), 231\left(100, \mathrm{M}^{+}-\mathrm{CH}_{3}\right), 216$ (15), $203\left(17, \mathrm{M}^{+}-\mathrm{CH}_{3}-\mathrm{CO}\right)$.

Aesculetin dimethyl ether $(5) . \mathrm{C}_{11} \mathrm{H}_{10} \mathrm{O}_{4}, \mathrm{mp} 145-146^{\circ} \mathrm{C}$. EIMS m/z (rel. Int.): 206 (100, $\left.\mathrm{M}^{+}\right), 191\left(36, \mathrm{M}^{+}-\mathrm{CH}_{3}\right), 178$ (21, $\left.\mathrm{M}^{+}-\mathrm{CO}\right), 163$ (44, $\left.\mathrm{M}^{+}-\mathrm{CH}_{3}-\mathrm{CO}\right), 135$ (42), 107 (43), 79 (52), 69 (74).

4-Methoxyphenylumbellate (6). $\mathrm{C}_{16} \mathrm{H}_{14} \mathrm{O}_{5}$, mp $169{ }^{\circ} \mathrm{C}$. $\mathrm{UV} \lambda_{\max } / \mathrm{nm}(\mathrm{MeOH}) 221,244,297,337 .{ }^{1} \mathrm{H}-$ and ${ }^{13} \mathrm{C}-\mathrm{NMR}$ (Table 1). EIMS $m / z$ (rel. Int.): $286\left(50, \mathrm{M}^{+}\right), 255$ (92, $\mathrm{M}^{+}-$ $\mathrm{OCH}_{3}$ ), 223 (56), 211 (55), 195 (42), 194 (57), 179 (31, $\mathrm{M}^{+}-\mathrm{C}_{7} \mathrm{H}_{7} \mathrm{O}$ ), 177 (68), 171 (37), 165 (58), 163 (52, M+ $\left.\mathrm{C}_{7} \mathrm{H}_{7} \mathrm{O}_{2}\right), 161$ (100). CIMS m/z (rel. Int.): $287\left(47, \mathrm{M}^{+}+1\right)$, $286\left(21, \mathrm{M}^{+}\right), 269$ (24), 255 (19), 213 (23), 179(27), 177 
(45), 163 (100). HRCIMS $m / z: 286.281\left(\mathrm{M}^{+}\right)$, calculated for $\mathrm{C}_{16} \mathrm{H}_{14} \mathrm{O}_{5} 286.282$.

\section{Synthesis of 4-Methoxyphenylumbellate (6)}

Preparation of Umbellic acid (7). $1 \mathrm{gm}$ of umbelliferone (Aldrich) was dissolved in $100 \mathrm{~mL} \mathrm{MeOH}$ and stirred with an equal volume of $5 \%$ alcoholic $\mathrm{KOH}$ for $4 \mathrm{~h}$. The reaction mixture was neutralized with diluted $\mathrm{HCl}$ and then extracted with EtOAc $(500 \mathrm{~mL})$. The residue left after evaporation of the solvent was purified over silica gel column $(100 \mathrm{~g}, 3$ cm) eluting with $\mathrm{CHCl}_{3}$ and $\mathrm{CHCl}_{3} / \mathrm{MeOH}$ mixtures. 455 $\mathrm{mg}$ of umbelliferone were recovered in the early fractions. Fractions 5- 11 eluted with $2 \% \mathrm{MeOH}$ in $\mathrm{CHCl}_{3}$ afforded $516 \mathrm{mg}$ of umbellic acid: $\mathrm{UV} \lambda_{\max } / \mathrm{nm}(\mathrm{MeOH}) 218,239$, 287, 323. ${ }^{1} \mathrm{H}-$ and ${ }^{13} \mathrm{C}-\mathrm{NMR}$ Table 1 . Found: C, 60.12; H, 4.61. Calc. for $\mathrm{C}_{9} \mathrm{H}_{8} \mathrm{O}_{4}$ (180.1): C, 60.00; $\mathrm{H}, 4.48$.

Protection of umbellic acid to $(7 \mathrm{a})$. To a solution of 7 (410 mg, $2.3 \mathrm{mmol}$ ) in $0.75 \mathrm{~mL}$ DMF, imidazol (938 mg, 13.8 mmol) and tert-butyldimethylsilylchloride (TBSCl) $(810 \mathrm{mg}$, $5.4 \mathrm{mmol}$ ) were added. The reaction mixture was stirred for 15 min under argon, quenched with $\mathrm{NaHCO}_{3}$, and stirring was continued for $5 \mathrm{~min}$. The resulted solution was extracted with $300 \mathrm{~mL}$ EtOAc. The organic layer was washed twice with $\mathrm{H}_{2} \mathrm{O}$, brine solution, then $\mathrm{H}_{2} \mathrm{O}$ again and finally dried over $\mathrm{Na}_{2} \mathrm{SO}_{4}$. Part of the residue left after evaporation of the EtOAc (7a) was checked by ${ }^{1} \mathrm{H}-\mathrm{NMR}$ and the rest was dried for the next step. UV $\lambda_{\max } / \mathrm{nm}\left(\mathrm{CHCl}_{3}\right) 228,241,293,325 .{ }^{1} \mathrm{H}-$ NMR Table 1. Found: C, 61.94; H, 8.79; Si, 13.91. Calc. for $\mathrm{C}_{21} \mathrm{H}_{36} \mathrm{O}_{4} \mathrm{Si}_{2}$ (408.6): C, 61.72; H, 8.88; Si, 13.74 .

Esterification of ( $7 \boldsymbol{a})$ to $(\boldsymbol{6 a})$. To a solution of $7 \mathbf{a}$ (165 $\mathrm{mg}, 0.49 \mathrm{mmol})$ in dry toluene, ECDI (141 mg, $0.74 \mathrm{mmol})$ and DMAP (90 mg, $0.74 \mathrm{mmol}$ ) were added. After stirring for $10 \mathrm{~min}$, a solution of $92 \mathrm{mg}$ 4-methoxyphenol $(0.74 \mathrm{mmol})$ in dry toluene was injected into the reaction solution and kept overnight at $55^{\circ} \mathrm{C}$ with stirring. The product of the reaction was purified by silica gel column $\left(30 \mathrm{~g}, 2 \mathrm{~cm}\right.$ ) eluted with $\mathrm{CHCl}_{3}$ to afford $97 \mathrm{mg}$ of $(\mathbf{6 a})$. UV $\lambda_{\max } / \mathrm{nm}(\mathrm{MeOH}) 241,287,296,330 .{ }^{1} \mathrm{H}-{ }^{13} \mathrm{C}-\mathrm{NMR}$ Table 1. Found: C, 65.40; H, 8.36; Si, 10.72. Calc. for $\mathrm{C}_{28} \mathrm{H}_{42} \mathrm{O}_{5} \mathrm{Si}_{2}$ (514.8): C, 65.32; H, 8.22; Si, 10.91.

Deprotection of (6a) to 4-Methoxyphenylumbellate (6). The protected ester $(\mathbf{6 a})(100 \mathrm{mg})$ was dissolved in dry THF, $2 \mathrm{~mL}$ of THF/pyridine were added and the solution was stirred at room temperature for $10 \mathrm{~min}$. The reaction mixture was extracted with $5 \% \mathrm{NaHCO}_{3}$, then $\mathrm{H}_{2} \mathrm{O}$. The organic layer after evaporation and prep TLC on silica gel afforded $45 \mathrm{mg}(\mathbf{6})$, which was identical with the isolated natural compound. HRCIMS $m / z: 286.280\left(\mathrm{M}^{+}\right)$, calculated for $\mathrm{C}_{16} \mathrm{H}_{14} \mathrm{O}_{5}$ 286.282.

\section{References}

1. Murry, R. D. H.; Mendez, J.; Brown, S. A.; The Natural Coumarins, Occurrence, Chemistry and Biochemistry, John Wiley\& Sons Ltd: Chichester, New York, Brisbane, Toronto, Singapore, 1982.

2. Okuyama, E.; Nishimura, S.; Ohmori, S.; Ozaki, Y.; Satake, M.; Yamazaki, M.; Chem Pharm. Bull. 1993, 41, 926.

3. Ulate-Rodriguez, J.; Schafer, H. W., Zottola, E. A.; Davidson, P. M.; J. Food Prod. 1997, 60, 1046.

4. Ulate-Rodriguez, J.; Schafer, H. W., Zottola, E. A.; Davidson, P. M.; J. Food Prod. 1997, 60, 1050.

5. Hudson, J. B.; Antiviral Compounds from Plants, CRC Press, Inc.: Boca Raton, Florida, 1990.

6. Ng, T. B., Huang, B.; Fong, W. P.; Yeung, H. W.; Life Sci. 1997, 61, 933.

7. Nivsarkar, M.; Desai, A.; Mokal, R.; Biochem. Mol. Biol. Int. 1996, 38, 625 .

8. Parrish, J. A.; Fitzpatrick, T. B.; Tanenbaum, L.; Pathak, M. A.; New Engl. J. Med. 1974, 291, 1207.

9. Boulos, L.; Flora of Egypt; Al Hadara Publishing, Cairo, Egypt, 2000, Vol. 2, p 167.

10. Ashkenazy, D.; Friedman, J.; Kashman, Y.; Planta Med. 1983, 47, 218.

11. Pouchert, C. J.; Behnke, J.; The Aldrich Library of ${ }^{13} \mathrm{C}$ and ${ }^{1} \mathrm{H}$ FTNMR Spectra, $1^{\text {st }}$ ed., Aldrich Chemical Company, Inc., USA, 1993.

12. Plattner, R. D.; Spencer, G. F.; Org. Mass. Spectrom. 1988, 23, 624.

13. Aplin R. T.; Page, C. B.; J. Chem. Soc. (C) 1967, 2593.

14. Mujumdar, A. S.; Usgaonkar R. N.; J. Chem. Soc., Perkin Trans 1 1974, 2236.

15. Kanvinde, M. N.; Kulkarni, S. A.; Paradkar, M. V.; Synth. Commun. 1990, 20, 3259.

16. Yamaguchi, K.; Spectral Data of Natural Products, Elsevier Publishing Co.: Amsterdam, London, New York, 1970, vol.1.

17. Anand, N. K.; Sharma, N. D.; Gupt, S. R.; Nat. Accad. Sci. Lett. (India) 1981, 4, 249.

18. Koshino, H.; Masaoka, Y.; Ichihara, A.; Phytochemistry 1993, 33, 1075

19. Linuma, M.; Matsuura, S.; Kusuda, K.; Chem. Pharm. Bull. 1980, 28, 708 .

20. Ayafor, J. F.; Connoly, J. D.; J. Chem. Soc., Perkin Trans. 1 1981, 2563.

21. Messana, I.; Ferrari, F.; Araujo M. D. M.; Planta Med. 1987, 6,541 .

22. Floss, H. G.; Mothes, U. Z.; Naturforsch. B 1964, 19, 770.

Received: October 8, 2001 Published on the web: November 14, 2002 